\title{
T cell hyperactivity in lupus as a consequence of hyperstimulatory antigen-presenting cells
}

\author{
JianKun Zhu, XueBin Liu, Chun Xie, Mei Yan, Ying Yu, Eric S. Sobel, \\ Edward K. Wakeland, and Chandra Mohan \\ Simmons Arthritis Research Center, Division of Rheumatology, Center for Immunology, \\ and University of Texas Southwestern Medical Center, Dallas, Texas, USA.
}

\begin{abstract}
Sle3 is an NZM2410-derived lupus susceptibility locus on murine chromosome 7. Congenic recombination has resulted in a novel mouse strain, B6.Sle3, associated with serum antinuclear autoantibodies (ANAs), T cell hyperactivity, and elevated CD4/CD8 ratios. An OVA-specific TCR transgene was used as a tool to demonstrate that Sle3 facilitated heightened $\mathrm{T}$ cell expansion in vitro, and in vivo, following antigen challenge. Indeed, continued $T$ cell expansion was noted even in response to a tolerogenic signal. However, these phenotypes did not appear to be $T$ cell intrinsic but were dictated by hyperstimulatory B6.Sle3 APCs. Importantly, B6.Sle3-derived DCs and macrophages appeared to be significantly more mature/activated, less apoptotic, and more proinflammatory and were better at costimulating T cells in vitro, compared with the $\mathrm{B} 6$ counterparts. Finally, the adoptive transfer of B6.Sle3-derived DCs into healthy $\mathrm{B} 6$ recipients elicited increased CD4/CD8 ratios and serum ANAs, 2 cardinal Sle3-associated phenotypes. We posit that their heightened expression of various costimulatory molecules, including CD80, CD106, I- $\mathrm{A}^{\mathrm{b}}$, and CD40, and their elevated production of various cytokines, including IL-12 and IL-1 $\beta$, may explain why Sle3-bearing DCs may be superior at breaching self tolerance. These studies provide mechanistic evidence indicating that intrinsic abnormalities in DCs and possibly other myeloid cells may dictate several of the phenotypes associated with systemic lupus, including ANA formation and T cell hyperactivity.
\end{abstract}

\section{Introduction}

$\mathrm{T}$ cells play a central role in driving the development of lupus, both in mice and in humans, as reviewed $(1,2)$. Earlier studies had indicated that the ablation of $\mathrm{CD} 4 \mathrm{~T}$ cells using genetic or experimental approaches had the potential to ameliorate disease (2-7). It is evident that $\mathrm{T}$ cells may be promoting disease in a variety of ways, including rendering help to B cells for autoantibody production, as well as facilitating tissue damage in the end organs (8-13). Although it is evident that $\mathrm{T}$ cells are essential for disease, the degree to which genetically dictated, intrinsic $\mathrm{T}$ cell anomalies contribute to lupus has not been clear.

In this respect, genetic dissection studies in murine models of lupus have contributed some insight. Spontaneously arising lupus in the NZM2410, NZB/NZW, and MRL/lpr strains is polygenic in origin, as reviewed (14-16). Interestingly, these different models share a disease susceptibility locus on mid-chromosome 7 (17-21). In the NZM2410 model, this locus has been termed Sle3. When this locus was introgressed onto the "normal" C57BL/6 (B6) background, it led to low levels of antinuclear autoantibodies (ANAs) and several lymphocyte phenotypes (22-24). Importantly, Sle3bearing $\mathrm{T}$ cells were spontaneously activated and exhibited elevated CD4/CD8 ratios and impaired activation-induced cell death (23). Although each individual locus by itself was not sufficient to engender severe lupus nephritis, epistatic interaction with other lupus susceptibility loci led to full-blown disease (25-27).

A surprise that next surfaced was the apparent cellular origin of the Sle3-associated phenotypes. When BM from allotype-marked B6

Nonstandard abbreviations used: ANA, antinuclear autoantibody; B6, C57BL/6; D, day.

Conflict of interest: The authors have declared that no conflict of interest exists.

Citation for this article: J. Clin. Invest. 115:1869-1878 (2005).

doi:10.1172/JCI23049. and B6.Sle 3 congenic mice were cotransferred into B6 hosts, T cells of both origins (i.e., with or without Sle3) exhibited elevated CD4/ $\mathrm{CD} 8$ ratios and spontaneous $\mathrm{T}$ cell activation, phenotypes attributed to Sle3 (28). These studies demonstrated that the Sle3-associated phenotypes may not be encoded in a $\mathrm{T}$ cell-intrinsic fashion, although they were BM-transferable. Likewise, the same study also revealed that autoantibody production in the chimeras was also not contingent upon the intrinsic expression of Sle3 within B cells (28).

The present study launches off from the above observations. An

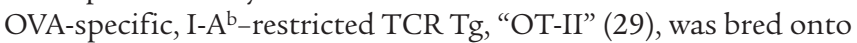
B6.Sle3 mice. B6.OT-II and B6.Sle3.OT-II mice were then examined to decipher how Sle3 may be impacting $\mathrm{T}$ cell activation and tolerance. In addition, the current study also elucidates the cellular players that may be intrinsically responsible for the $\mathrm{T}$ cell hyperactivity attributed to Sle3. Importantly, this study draws attention to the potential role of nonlymphocytic myeloid-lineage cells in driving systemic autoimmunity in lupus.

\section{Results}

Phenotypes of novel congenic/transgenic mice. As summarized in Table 1, B6.Sle3 mice (without the Sles locus) exhibited elevated CD4/CD8 ratios, as well as the serological and pathological phenotypes previously described in B6.Sle3/Sle5 congenics $(22,23)$. To further study the cellular origins of these phenotypes, the OVA-specific TCR Tg, OT-II (29), was successfully bred onto B6.Sle3 mice. As observed previously in B6.Sle3 congenics (22), B6.Sle3.OT-II mice also possessed low but significant levels of ANAs (Figure 1A), but not glomerulonephritis (data not shown). B6.OT-II and B6.Sle3.OT-II $\mathrm{Tg}$ mice exhibited similar numbers of $\mathrm{T}$ cells and $\mathrm{B}$ cells in their spleens and nodes (data not shown). Importantly, the B6.OT-II and B6.Sle3.OT-II Tg mice expressed both the $V \beta 5$ and the $V \alpha 2$ TCR transgenes on about $75 \%$ of their peripheral $\mathrm{CD} 4^{+} \mathrm{T}$ cells (Figure 1B). Ex vivo, B6.Sle3.OT-II TCR Tg T cells were not more 
Table 1

Phenotypes of B6.Sle3 and B6.Sle3/Sle5 congenics, and OT-II transgenics

$\begin{array}{lccc} & \text { B6 } & \text { B6.Sle3/5 } & \text { B6.Sle3 } \\ \text { Genetic background } & \text { B6 } & \text { B6 } & \text { B6 } \\ \text { Number examined } & 12 & 12-22 & 11-12 \\ \text { Reference } & & 22,23 & \text { Present report } \\ \text { Congenic loci } & \text { None } & \text { Sle3 and Sle5 } & \text { Sle3 } \\ \text { Minimal NZM interval } & 0 & 40 \mathrm{cM} & 24 \mathrm{cM} \\ \text { Delimiting markers } & & \text { D7mit56-D7mit40 } & \text { D7mit158-D7mit40 } \\ \text { Phenotypes } & & & \\ \text { Splenic CD4/CD8 ratios } & 1.5 \pm 0.09 & 2.4 \pm 0.19^{\mathrm{B}} & 2.1 \pm 0.15^{\mathrm{A}} \\ \text { IgG anti-ssDNA (U/ml) } & 8.2 \pm 0.9 & 36.4 \pm 6.0^{\mathrm{B}} & 28.6 \pm 3.1^{\mathrm{B}} \\ \text { IgG anti-dsDNA (U/ml) } & 3.8 \pm 0.9 & 19.6 \pm 3.3^{\mathrm{B}} & 16.4 \pm 2.3^{\mathrm{B}} \\ \text { \% Grade 3 or 4 GN } & 0 \% & 18 \% & 27 \%\end{array}$

The phenotypes of B6 mice, B6.S/e3 congenics, and B6.Sle3/Sle5 congenics (possessing a single interval spanning Sle3 and Sle5) were compared at 9-12 months of age. Since ANAs do not become prominent until the mice are aged $(22,25,26)$, all mice were examined at 9-12 months of age for serological phenotypes and pathology. Whereas the CD4/CD8 ratios were determined using flow cytometry, serum ANAs were assayed using ELISA, as previously published (22, 25, 26). Phenotype mean \pm SEM is shown, comparing the congenics with the B6 controls, using the Student's $t$ test. The corresponding values of these same phenotypes in related polycongenic strains and the NZM2410 parental strain are detailed elsewhere (25, 26). ${ }^{A} P<0.01$; ${ }^{B} P<0.001$. ssDNA, single-stranded DNA; dsDNA, double-stranded $\mathrm{DNA} ; \mathrm{GN}$, glomerulonephritis.

activated (based on CD69 expression), compared with B6.OT-II TCR Tg T cells (Figure 1C).

Sle3 impacts $T$ cell function: in vitro and in vivo studies. Although B6.OT-II and B6.Sle3.OT-II mice exhibited similar numbers of Tg $\mathrm{T}$ cells, Sle3-bearing $\mathrm{Tg} \mathrm{T}$ cells were hyperproliferative, as displayed in Figure 2A, with an attendant reduction in activation-induced cell death (data not plotted), consistent with previous findings (23). Previous studies had also shown that the impact of Sle 3 on T cells was not dependent on T cell-intrinsic Sle3 expression (28). Hence, we designed an in vivo adoptive transfer experiment to confirm this, using the OT-II TCR Tg model. In essence, B6.OT-II Tg T cells were adoptively transferred into $\mathrm{B} 6$ or B6.Sle3 hosts, after which the recipient mice were challenged with an immunogenic dose of OVA. In resonance with the earlier report (28), the administration of immunogenic OVA led to a greater degree of $\mathrm{T}$ cell expansion in vivo in B6.Sle3 hosts, with an attendant increase in serum IgG anti-OVA Abs (Figure 2B). After challenge with OVA, T cells in both types of hosts were equally activated ( $>90 \%$ of Tg T cells expressed CD69; data not plotted). Finally, Sle3-bearing T cells appeared relatively recalcitrant to peripheral tolerance induction, as depicted in Figure 2, C and D. Thus, when B6.OT-II mice were first exposed to their cognate anti-

\section{Figure 1}

Serological and cellular phenotypes in B6.Sle3.OT-II mice. (A) Serum levels of antinuclear autoantibodies were assessed in 9- to 12-monthold B6.OT-II and B6.Sle3.OT-II mice ( $n=8$ mice per group). The dashed line indicates the mean serum levels of ANAs in B6.Sle1.FAS'pr control mice exhibiting full-blown lupus (27). (B and $\mathbf{C}$ ) The spleens of 3- to 6-month-old B6.OT-II and B6.Sle3.OT-II mice (9-12 mice per group) were also examined for the prevalence of $\mathrm{V}_{3} 5^{+}, \mathrm{V \alpha 2}^{+}$, and $\mathrm{CD} 4^{+}$transgenic $\mathrm{T}$ cells (B), and their activation status, based on the surface expression of CD69 (C). Spleens of both strains did not differ in their total numbers of splenocytes, or splenic CD4 T cells. Mean \pm SEM is shown in all plots. The depicted $P$ values were derived using the Student's $t$ test.

gen under a tolerizing regime, their T cells were not able to mount a proliferative response when subsequently challenged with an immunogenic form of OVA. In contrast, the presence of Sle 3 abrogated effective peripheral tolerance in this experimental model (Figure 2D).

Sle3 encodes aberrant myeloid-lineage cells. Given that previous allotype-marked BM transfer experiments (28) and the adoptive transfer studies described above (Figure $2 \mathrm{~B}$ ) indicated that the Sle3-associated phenotypes may not be $\mathrm{T}$ cell intrinsic, we asked whether quantitative or qualitative differences in Sle3-bearing cells of myeloid origin, including various APC subsets, may be responsible for the phenotypic differences noted above. Spleens, lymph nodes, and peripheral blood from 9- to 12-month-old B6 and B6.Sle3 mice were examined for the numbers and phenotypes of DCs, macrophages, and neutrophils, by flow cytometry, as illustrated in Figure 3 , A and B. As noted in Table 2, myeloid-lineage cells from B6.Sle3 spleens demonstrated several quantitative and qualitative differences. The most consistent difference was the expanded percentages of macrophages in 9- to 12-month-old B6.Sle3 spleens, which was noted in multiple experiments (Table 2). Since B6 and B6.Sle3 mice possessed similar numbers of splenocytes, these differences in percentages also translated to differences in the absolute numbers of these cells. Although splenic neutrophils were also expanded in numbers, these differences fell short of statistical significance. There were no significant differences in the numbers of splenic myeloid DCs, though the numbers of lymphoid DCs and plasmacytoid DCs tended to be relatively higher (Table 2, and data not shown).

In addition, DCs, macrophages, and neutrophils isolated from B6.Sle3 spleens were evidently more activated/mature, based on the expression of several surface markers (Table 2). Thus, for example, although the myeloid DCs were not expanded in numbers in B6.Sle3 spleens, they exhibited increased surface levels of CD40, CD80, CD86, CD54, CD106 (VCAM-1), and FcR (CD16/32), with similar differences being noted on B6.Sle3 lymphoid DCs, macrophages, and neutrophils (Table 2). In particular, the surface levels of CD106 were about twice as high on all 4 cell subsets examined, compared with the corresponding levels in B6-derived cells. Similar changes were also noted in the lymph nodes and peripheral blood of B6.Sle 3 mice (Table 3, and data not shown). Interestingly,

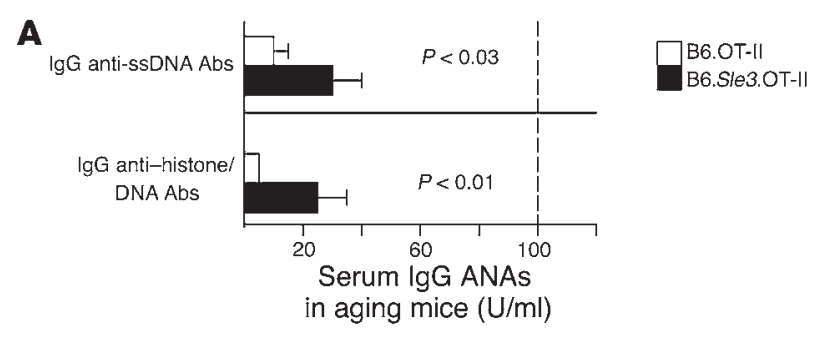

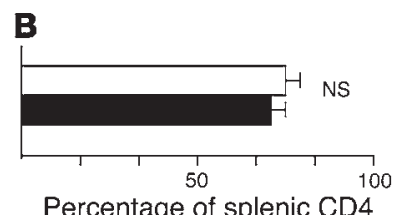

Percentage of splenic CD4

$\mathrm{T}$ cells that possessed the $\mathrm{Tg}$ c

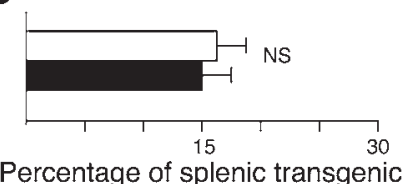

$T$ cells that were CD69+ 
B6.Sle3 lymph node-derived DCs, macrophages, and neutrophils displayed CD40 and I-A $A^{b}$ levels that were severalfold higher than the respective expression levels on the $\mathrm{B} 6$ controls.

As noted above in the spleens and nodes of older B6.Sle 3 mice, one could also discern the heightened activation of different myeloid-lineage cells in young (i.e., 2-month-old) B6.Sle3 mice (Figure 3, C and D). Likewise, macrophages cultured from B6.Sle3 BM (using M-CSF) showed similar phenotypic differences, with these differences becoming amplified following LPS stimulation (Figure 3E). This was particularly pronounced with respect to CD80 expression. B6.Sle3-derived BM-cultured DCs revealed similar phenotypic differences, when the DCs were elicited using GM-CSF (Figure 3F). On the other hand, DCs that were cultivated using GM-CSF plus IL-4 yielded mixed results: B6-derived DCs appeared to be more activated in 2 experiments, the inverse pattern was noted in a third experiment, and no differences were noted in a fourth experiment (data not shown). The reason for this variability with DCs elicited by GM-CSF plus IL-4 is not presently clear.

Sle3 myeloid cells exhibit altered cytokine profiles and impaired apoptosis. B6.Sle3-derived macrophages and DCs also exhibited altered cytokine production profiles, compared with the $\mathrm{B} 6$ controls, as displayed in Figure 4. Unmanipulated splenic DCs isolated from B6.Sle3 congenics, as well as BM-derived DCs (cultured using GM-CSF), produced more IL- 12 but less IL- 6 in culture (Figure 4, A and B). In addition, B6.Sle3 DCs also hypersecreted IL-1 $\beta$ and showed a variable difference in TNF- $\alpha$ production (Figure 4B; and see Supplemental Figure 1; supplemental material available online with this article; doi:10.1172/JCI23049DS1). Although IL-12, IL-6, and TNF- $\alpha$ were elevated in B6.Sle3 macrophage supernatant, IL-1 $\beta$ secretion was variably increased (Figure 4, C-E, and Supplemental Figure 1).

Besides the elevated production of proinflammatory cytokines, B6.Sle3-derived myeloid cells also revealed another interesting phenotypic difference - impaired apoptosis (Figure 5, A and B). This difference was demonstrated for both BM-derived DCs and macrophages (Figure 5, A and B). Stimulation with LPS did not alter this phenotypic difference (data not shown). These findings are in line with our recent observation that B6.Sle 3 mice also exhibit reduced apoptosis of neutrophils in vitro and in vivo, in an infection challenge model (B. Mehrad and C. Mohan, manuscript submitted for publication). However, Sle3 did not impact the phagocytic poten-
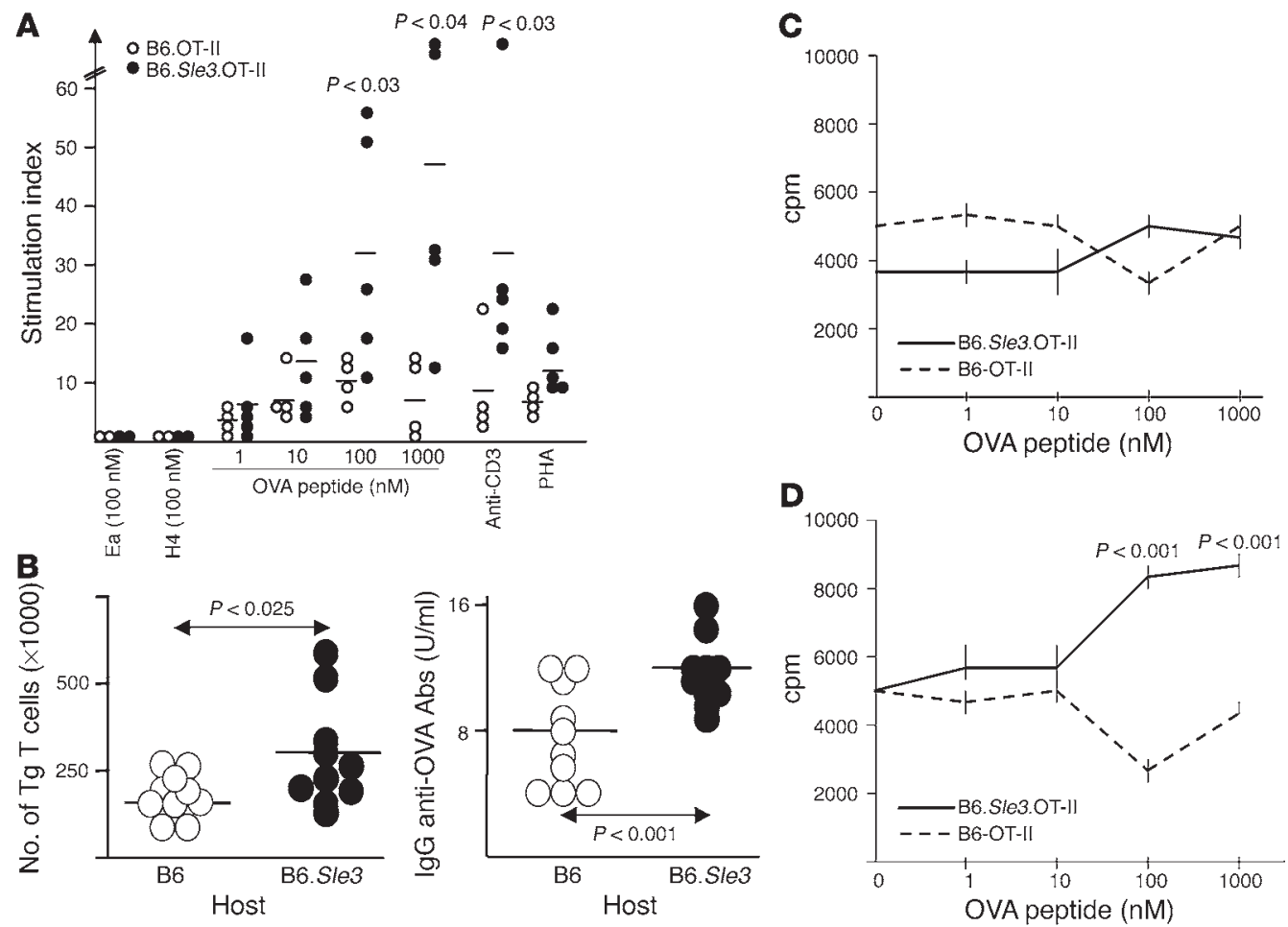

Figure 2

Functional responsiveness of B6.Sle3.OT-II T cells. (A) B6.OT-II and B6.Sle3.OT-II splenocytes were cultured with various stimuli and assessed for proliferation. Stimulation index was $\mathrm{cpm}_{\text {experiment }} / \mathrm{cpm}_{\text {no-antigen. The }} \mathrm{cpm}_{\text {no-antigen }}$ values ranged from 2,000 to 5,000. Each dot represents an independent spleen sample. Horizontal bars indicate the respective group means. The data shown were reproduced in 2 additional studies (Supplemental Table 1 [supplemental material available online with this article; doi:10.1172/JCI23049DS1]). (B) B6.OT-II splenic T cells were transferred i.v. into 2-month-old B6 or B6.Sle3 mice on D0 and challenged with an immunogenic form of OVA on D1, as described in Methods. Seven days after transfer, the numbers of $\mathrm{Tg} \mathrm{T}$ cells in the recipient spleens (left) and serum IgG anti-OVA levels (right) were assessed. Horizontal bars indicate group means. The displayed data were pooled from 2 independent studies using 5 mice per strain. (C) B6.OT-II and B6.Sle3. OT-II mice ( $n=5$ per group) were challenged with OVA O $23-339_{1}$ in incomplete Freund's adjuvant on D0; splenocytes were isolated on D5 and assessed for their proliferative response to OVA $323-339$. The vertical bars represent the SEM of triplicate cultures. Data shown are representative of 2 independent studies. Observed differences were not statistically significant. (D) B6.OT-II and B6.Sle3.OT-II mice ( $n=5$ mice per group) were challenged first with tolerogenic OVA on D0 and then with immunogenic OVA on D10 and examined as described in Methods. The vertical bars represent the SEM of triplicate cultures. In a second confirmatory study (data not shown), the fold difference in cpm between the 2 strain groups was $1.9(P<0.04, n=4$ each), at an OVA stimulation dose of $1,000 \mathrm{nM}$. 

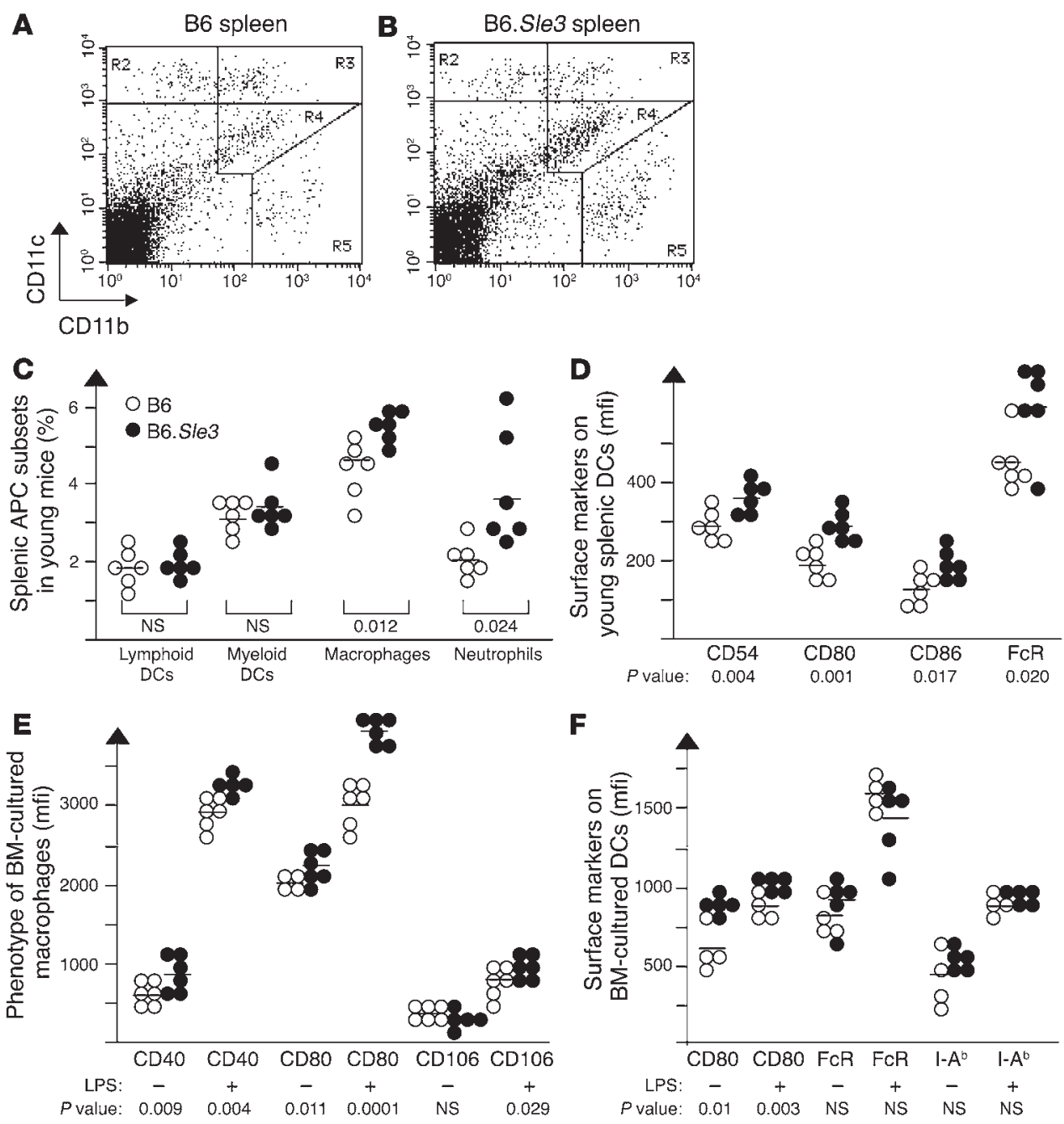

Figure 3

Splenic and BM myeloid cell subsets in B6 and B6.Sle3 mice. (A and B) Representative CD11b/CD11C expression profiles observed in 9- to 12-month-old B6 and B6.Sle3 collagenase-treated spleens. The cells in region R2, also expressing CD8, were gated as lymphoid DCs, whereas the cells in region R3 were gated as myeloid DCs. Cells that were in region R4 were gated as macrophages; these cells also exhibited high side scatter and F4/80 expression. Cells in region R5 were gated as neutrophils, and these cells also exhibited high Gr-1 levels. The prevalence of these cells in the spleens of 9- to 12-month-old B6 and B6.Sle3 mice ( $n=10-15$ each), and the expression levels of CD40, CD80, CD106 (VCAM-1), and FcR on these 4 gated populations, are detailed and statistically compared in Table 2. (C) The respective percentages of the different myeloid cell populations in 2-month-old B6 and B6.Sle3 spleens. (D-F) Expression levels (mean fluorescence intensities, $\mathrm{mfi}$ ) of various surface markers on 2-month-old splenic CD11 b $\mathrm{b}^{+++}$, CD11 $\mathrm{C}^{+++}$myeloid DCs (D); on BM M-CSF-cultured macrophages, gated on $\mathrm{F} 4 / 80^{+}$cells (E); and on BM GM-CSF-cultured DCs, gated on CD11 $\mathrm{C}^{+}$cells $(\mathbf{F})$. For the latter 2 studies, the cells were phenotyped with or without LPS pretreatment $(10 \mathrm{ng} / \mathrm{ml}, 24$ hours). For all studies, each dot represents data derived from an individual mouse. Data are representative of 2-3 independent studies each. Depicted $P$ values were computed by comparison of the B6.Sle3 values with the B6 control values, using the Student's $t$ test. The horizontal bars indicate the respective group means.

tial of macrophages (B. Mehrad and C. Mohan, manuscript submitted for publication). Interestingly, when Sle3-bearing DCs were OVA-pulsed and cocultured with OT-II TCR Tg T cells, the T cells also demonstrated reduced apoptosis with a concomitant increase in activation, as gauged by the surface expression of CD69 (Figure 5, $\mathrm{C}$ and $\mathrm{D})$, compared with $\mathrm{T}$ cells cocultured with B6 DCs.

Sle3 APCs are superior at costimulating $T$ cells. In keeping with the above findings, the more activated/mature phenotype of B6.Sle3- derived DCs was also accompanied by an enhanced $\mathrm{T}$ cell costimulatory capacity. When BM-derived DCs or splenic DCs were cocultured with OVA-reactive B6.OT-II TCR Tg T cells, Sle3-bearing DCs induced a greater degree of $\mathrm{T}$ cell division and proliferation, in a manner dependent on antigen dose and APC number (Figure 6). Similar results were noted when $T$ cell alloresponsiveness was assayed (data not shown). Although the difference in thymidine incorporation attained statistical significance, the difference in cell division (as assayed by CFSE dilution) failed to attain significance within individual experiments. In both the antigen-specific response experiments and the alloresponse studies, the use of high numbers of B6-derived DCs tended to dampen the proliferative response of the responding $\mathrm{T}$ cells (Figure $6, F$ and $G$, and data not shown); in contrast, Sle3-bearing DCs continued to be hyperstimulatory even when high numbers of DCs were used for costimulation.

These differences were most apparent when B6.OT-II TCR Tg $\mathrm{T}$ cells were used for the cocultures; B6.Sle3.OT-II Tg T cells behaved fairly similarly irrespective of whether B6 or B6.Sle3 DCs were used for stimulation (Figure $6, \mathrm{E}$ and $\mathrm{H})$. In all experiments, the extent of cell division of the $\mathrm{Tg} \mathrm{T}$ cells failed to approximate $100 \%$, possibly because of the expression of non-Tg TCR since these studies were executed on a $R A G$-sufficient background. Finally, T cells cocultured with B6.Sle3 DCs were demonstrated to produce more IFN- $\gamma$, compared with $\mathrm{T}$ cells cocultured with B6-derived DCs, in some experiments but not others (data not shown), consistent with the elevated IL-12 production by B6.Sle3 DCs.

Sle3 DCs recapitulate the in vivo phenotypes attributed to Sle3. Given that the Sle 3 locus facilitated the generation of APCs that were evidently more mature, proinflammatory, and costimulatory, we next asked whether this cellular phenotype might indeed be responsible for the $\mathrm{T}$ cell and serological phenotypes noted in B6.Sle3 mice. Importantly, the adoptive transfer of Sle3-bearing DCs (compared with B6-derived DCs) into young B6 hosts, when coupled with LPS coadministration, led to elevated splenic CD4/CD8 ratios (Figure 
7A) and elevated serum autoantibody levels (Figure 7B), 2 cardinal Sle3-associated phenotypes $(22,23)$. In these adoptive transfer studies, the maximal autoantibody levels were noted on day 40 (D40), i.e., about 4 weeks after the last administration of DCs (see legend to Figure 7). However, this serological outcome was not sufficient for renal disease to ensue (data not shown). The transfer of DCs alone, without any added LPS, or the administration of LPS alone, was insufficient to elicit the above phenotypes (data not shown). These studies demonstrate that Sle3-bearing DCs appear to be sufficient to recreate the Sle3-associated immunophenotypes.

\section{Discussion}

Sle3 is an NZM2410/NZW-derived locus on proximal chromosome 7 that facilitates spontaneous $\mathrm{T}$ cell hyperactivity and low-grade serological autoreactivity, when introgressed onto the normal B6 genetic background $(22,23)$. The present report adds to the earlier findings in several respects. Firstly, whereas the earlier report had detailed the phenotypic properties of a $40-\mathrm{cM}$ interval on proximal chromosome 7 encompassing Sle3 and Sle5, the present report focuses on mice that bear a 24-cM interval on mid-chromosome 7, encompassing Sle3 but not Sle5 (Table 1 and Figure 1). Secondly, the $\mathrm{T}$ cell repertoire is greatly simplified in the current study, owing to the use of an OVA-specific TCR Tg. Finally, and most importantly, the present study directs attention to the potential importance of aberrant myeloid-lineage cells in contributing to the immunological phenotypes associated with this congenic interval. Importantly, the adoptive transfer experiments indicate that the genetic makeup of the APC has the potential to influence the degree of T cell hyperactivity as well as serological autoreactivity, in lupus pathogenesis.

The current findings fortify the conclusions of several previous reports implicating the potential role of enhanced and/or hyperactive APCs in driving autoimmunity. Although the most cited example of elevated monocytosis in murine lupus is the BXSB model (30-32), increased macrophages have also been documented in BWF1 and MRL/lpr lupus mice $(33,34)$, with attendant anomalies in cytokine profiles (35). Likewise, aberrant DC function has also been reported in murine lupus (36-38), as well as the NOD model of diabetes (39-42). Additionally, forward genetic studies have generated several "engineered" models of disease where systemic autoimmunity and myeloid hyperactivity both co-dominate the clinical phenotype. Thus, for example, Shp-1, lyn CD200/OX2, rel-B, and Nfkb2 mice all have the potential to influence both autoimmunity and the myeloid cell compartment (43-47). Although Sle3-bearing APCs resemble APCs from mouse strains exhibiting aberrant expression of the above molecules, Sle 3 does not represent an allelic variant of any of the above genes, as they are located on different chromosomes.

The notion that aberrant APCs can breach T cell tolerance is well accepted (48). The underlying mechanism(s) that may explain why Sle3-bearing APCs may be more potent at stimulating T cells in vitro, or in breaching tolerance in vivo, is an important issue to investigate. On the one hand, Sle3-bearing DCs (and other APCs) may be more "costimulatory," because of their increased surface expression of CD80/B7-1, I-A b CD40, etc. The observation that blockade of B7:CD28 and CD40:CD40L interactions has the potential to ameliorate murine lupus lends support to this thesis (49-51). In addition, their heightened secretion of proinflammatory cytokines may further augment the costimulatory potential of Sle3 APCs. The notion that a proinflammatory milieu could promote the rapid maturation of DCs and impair their T cell-tolerizing poten- tial is well documented (52-55). Clearly, both of the above mechanisms (i.e., increased costimulation and proinflammatory properties) may be synergistic in breaching $\mathrm{T}$ cell tolerance, as has been expounded by others $(55,56)$. Although one might have anticipated a phenotypic difference when Sle3-bearing DCs were adoptively transferred into a B6 host, even without LPS coadministration, this was not the case. Apparently, the coadministration of LPS was essential to uncover the autoimmune potential of B6.Sle3 DCs. It is conceivable that LPS stimulation of the transferred B6.Sle3 DCs may be required for maximally modulating their activation status, cytokine profile, or survival advantage in vivo, drawing from the in vitro observations presented in Figures 3-5.

Based on the in vitro studies in Figure 5, we postulate that the more mature and proinflammatory nature of B6.Sle3 DCs may have conferred a stronger proliferative edge and a better survival advantage to potentially autoreactive CD4 T cells. This could also

\section{Table 2}

Phenotype of DCs, macrophages, and neutrophils in B6 and B6.Sle3 spleens

\begin{tabular}{|c|c|c|c|}
\hline & B6 & B6.Sle3 & $P$ value ${ }^{A}$ \\
\hline \multicolumn{4}{|l|}{ Lymphoid DCs } \\
\hline$\%$ in spleen $\mathrm{B}$ & $1.8 \pm 0.3$ & $2.4 \pm 0.5$ & 0.048 \\
\hline CD40 mfic & $291 \pm 57$ & $395 \pm 39$ & 0.027 \\
\hline CD106 mfic & $628 \pm 93$ & $1,010 \pm 173$ & 0.03 \\
\hline CD80/B7-1 mfiD & $724 \pm 33$ & $1,073 \pm 103$ & 0.006 \\
\hline CD54 mfi & $451 \pm 12$ & $505 \pm 23$ & 0.038 \\
\hline FcR mfic & $743 \pm 67$ & $942 \pm 67$ & 0.005 \\
\hline \multicolumn{4}{|l|}{ Myeloid DCs } \\
\hline$\%$ in spleen $B$ & $2.9 \pm 0.5$ & $2.7 \pm 0.5$ & NS \\
\hline CD40 mfic & $204 \pm 22$ & $337 \pm 76$ & 0.005 \\
\hline CD106 mfic & $455 \pm 89$ & $936 \pm 249$ & 0.003 \\
\hline CD80/B7-1 mfic & $739 \pm 97$ & $1,204 \pm 170$ & 0.015 \\
\hline CD86/B7-2 mfic & $60 \pm 13$ & $128 \pm 33$ & 0.002 \\
\hline CD54 mfic & $254 \pm 25$ & $339 \pm 35$ & 0.007 \\
\hline FcR mfic & $526 \pm 56$ & $831 \pm 104$ & 0.0003 \\
\hline \multicolumn{4}{|l|}{ Macrophages } \\
\hline$\%$ in spleen $\mathrm{B}$ & $5.3 \pm 0.8$ & $6.8 \pm 0.9$ & 0.026 \\
\hline CD40 mfic & $159 \pm 33$ & $262 \pm 67$ & 0.014 \\
\hline CD106 mfiD & $609 \pm 133$ & $1,459 \pm 331$ & 0.040 \\
\hline B7-1 mfi & $1,210 \pm 49$ & $1,382 \pm 108$ & 0.026 \\
\hline \multicolumn{4}{|l|}{ Neutrophils } \\
\hline$\%$ in spleen ${ }^{B}$ & $3.8 \pm 1.2$ & $5.5 \pm 2.4$ & NS \\
\hline CD40 mfic & $62 \pm 11$ & $99 \pm 31$ & 0.036 \\
\hline CD106 mfic & $401 \pm 39$ & $690 \pm 83$ & 0.0002 \\
\hline CD80/B7-1 mfic & $147 \pm 52$ & $288 \pm 119$ & 0.024 \\
\hline $\mathrm{FcR} \mathrm{mfjC}$ & $377 \pm 37$ & $449 \pm 47$ & 0.05 \\
\hline
\end{tabular}

AP values pertain to Student's $t$ test statistical comparisons of B6 versus B6.Sle3 cells isolated from collagenase- and DNase I-treated spleens of 9- to 12-month-old mice. Only surface markers that were differentially expressed on the respective cell populations are listed. BData represent mean \pm SEM pertaining to $15 \mathrm{~B} 6$ samples and $15 \mathrm{~B} 6$. Sle3 samples. Lymphoid DCs were CD11 $\mathrm{c}^{+++}$and $\mathrm{CD} 8^{++}$, but CD11 $\mathrm{b}^{\text {intermediate }}$ (CD11 bint). Myeloid DCs were CD11 $\mathrm{c}^{+++}$and CD11b $\mathrm{b}^{+++}$but CD8 ${ }^{\text {low }}$, and

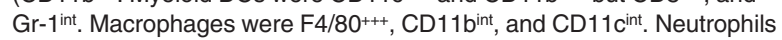
were $\mathrm{Gr}-1^{+++}$and $\mathrm{CD} 11 \mathrm{~b}^{+++}$, but $\mathrm{CD} 11 \mathrm{c}^{-}$, and F4/80 int. CData were obtained by examination of 10-15 mice per strain and are representative of at least 2 independent FACS-staining experiments involving 5 B6 mice and 5 B6.Sle3 mice each. Depicted staining intensities were significantly higher than the isotype-control staining, as detailed in Methods. DData pertain to a single FACS-staining experiment involving 5 B6 mice and 5 B6.Sle 3 mice but were not reproduced in a second FACS-staining experiment. mfi, mean fluorescence intensity. 


\section{Table 3}

Phenotype of DCs, macrophages, and neutrophils in B6 and B6.Sle3 lymph nodes

\begin{tabular}{|c|c|c|c|}
\hline & B6 & B6.Sle3 & $P$ value ${ }^{A}$ \\
\hline \multicolumn{4}{|l|}{ Lymphoid DCs } \\
\hline$\%$ in lymph node & $3.3 \pm 0.6$ & $3.2 \pm 0.7$ & NS \\
\hline CD40 mfic & $1,129 \pm 151$ & $1,740 \pm 283$ & 0.001 \\
\hline I-Ab mfiD & $864 \pm 55$ & $1,560 \pm 226$ & 0.008 \\
\hline \multicolumn{4}{|l|}{ Myeloid DCs } \\
\hline$\%$ in lymph node & $4.2 \pm 2.0$ & $5.2 \pm 2.1$ & NS \\
\hline CD40 mfic & $720 \pm 150$ & $1,325 \pm 350$ & 0.006 \\
\hline CD54 mfiD & $598 \pm 22$ & $734 \pm 30$ & 0.008 \\
\hline FcR mfiC & $795 \pm 156$ & $1,153 \pm 241$ & 0.050 \\
\hline \multicolumn{4}{|l|}{ Macrophages } \\
\hline$\%$ in lymph node & $6.2 \pm 1.5$ & $9.7 \pm 2.2$ & NS \\
\hline CD40 mfic & $382 \pm 112$ & $909 \pm 311$ & 0.023 \\
\hline I-Ab mfic & $247 \pm 55$ & $525 \pm 145$ & 0.010 \\
\hline FcR mfiD & $102 \pm 21$ & $192 \pm 26$ & 0.032 \\
\hline \multicolumn{4}{|l|}{ Neutrophils } \\
\hline$\%$ in lymph node & $1.6 \pm 0.5$ & $3.2 \pm 0.9$ & 0.007 \\
\hline CD40 mfiD & $124 \pm 32$ & $1,335 \pm 3,829$ & 0.034 \\
\hline CD106 mfiD & $411 \pm 59$ & $1,091 \pm 116$ & 0.0002 \\
\hline $\mathrm{I}-\mathrm{A}^{\mathrm{b}} \mathrm{mfi}$ & $182 \pm 76$ & $404 \pm 62$ & 0.002 \\
\hline CD80/B7-1 mfiD & $378 \pm 88$ & $742 \pm 57$ & 0.008 \\
\hline CD54 mfiD & $113 \pm 16$ & $204 \pm 58$ & 0.008 \\
\hline FcR mfic & $459 \pm 58$ & $772 \pm 118$ & 0.002 \\
\hline \multicolumn{4}{|c|}{ 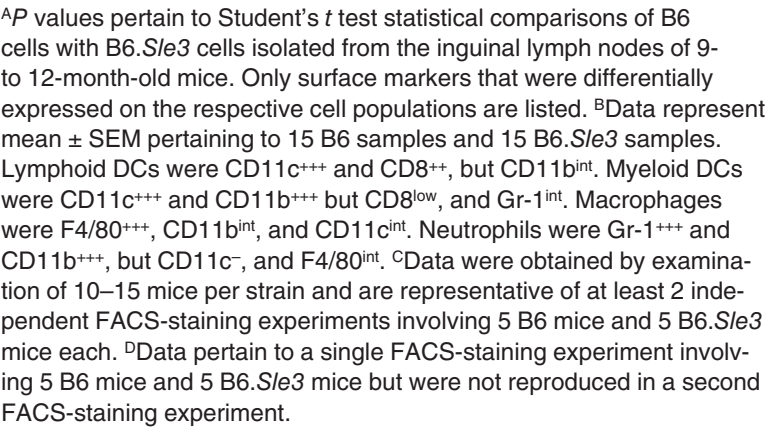 } \\
\hline
\end{tabular}

account for the increased CD4/CD8 ratios noted in unmanipulated B6.Sle 3 mice (23), and in the adoptive transfer experiments (Figure 7). Autoreactive members among the expanded pool of CD4 T cells may in turn have been responsible for the increased serum ANAs observed, both in unmanipulated B6.Sle 3 congenics $(22,23)$ and in the adoptive transfer studies (Figure 7), through T-dependent B cell help. However, a direct impact of Sle3-bearing myeloid cells on B cell function cannot yet be excluded. In addition to their potential impact on systemic T and B cells, it is conceivable that the hyperactive B6.Sle3 myeloid cells may also be responsible for the enhanced susceptibility to renal disease seen in B6.Sle3 congenics (Y. Fu and C. Mohan, unpublished observations). The notion that neutrophils and macrophages play an essential role in immune-mediated renal disease is well accepted $(57,58)$, and these cells do appear to play a more prominent role in the renal disease seen in B6.Sle3 mice (Y. Fu and C. Mohan, unpublished observations).

Collectively, it appears likely that the hyperactivated myeloid compartment in B6.Sle3 mice may be contributing to lupus through multiple mechanisms, both systemically and locally in the end organs. Although the expression of Sle 3 within the myeloid cell compartment of these congenics appears to be sufficient to elicit the previously described Sle3-associated phenotypes, one cannot exclude the possibility that Sle 3 may also be impacting additional cell types, and these additional cellular mechanisms may also be contributing to lupus pathogenesis. Specifically, one cannot exclude the possibility that T cell-intrinsic expression of Sle3 may be contributing at least in part to the "T cell hyperresponsiveness" noted in these mice. Although the T:DC coculture studies portrayed in Figure 6E might support this thesis, it is clearly possible that $\mathrm{T}$ cells isolated from B6.Sle3 congenics may appear to be hyperresponsive simply because they might have already been "primed" by Sle3-bearing APCs in vivo.

The chromosome 7 interval encompassing Sle 3 has also been implicated in genetic analyses of other lupus models (18-21, 59). Presently, it is not clear whether the different loci uncovered in the different mouse models represent allelic variants of the same culprit gene(s). Given the repeated mapping of this chromosomal interval in several independent murine lupus studies and models, and the intriguing phenotypes associated with this locus, elucidating the candidate genes within this locus is of paramount importance. Since the phenotypes exhibited by the B6.Sle3-derived macrophages, DCs, neutrophils, and $\mathrm{T}$ cells are fundamentally similar (i.e., reduced apoptosis with a concomitant increase in activation status), we hypothesize that a single genetic defect may be responsible for all of these cellular phenotypes. On the other hand, since the studied congenic interval is fairly large, it is certainly possible that 2 or more genes within the disease interval may be contributing to the observed cellular phenotypes. Among the 560 genes/open reading frames located within the Sle 3 congenic interval, 1 gene that had potential significance to APC biology was FL (Flt3 ligand). However, sequencing and expression studies of the FL gene in $\mathrm{B} 6$ and B6.Sle 3 mice have failed to substantiate FL as the culprit gene for Sle3. Progressive narrowing of the recombinant congenic interval and additional candidate gene testing are in progress.

Given that Sle 3 is a major disease susceptibility locus in the NZM2410 lupus model, one may infer that the " $\mathrm{T}$ cell hyperactivity" in NZM2410 lupus mice may be contingent upon intrinsic APC anomalies, to a significant degree. Lupus $T$ cells have also been phenotyped as being hyperactive in other murine models, as reviewed $(1,2)$. Based on the present findings, it becomes important to evaluate the extent to which more exuberant APC function may also underlie the T cell hyperactivity noted in other lupus models. Finally, $\mathrm{T}$ cell hyperactivity, including several biochemical abnormalities that correlate with $\mathrm{T}$ cell hyperresponsiveness, and aberrant DC function have also been documented in human lupus (1, 60-67). The current findings raise the possibility that the $T$ cell phenotypes noted in human SLE may also be influenced by intrinsic differences in DC/myeloid cell function, in addition to possible T cell-intrinsic anomalies (65-67). Focusing future research efforts on the myeloid compartment may not only shed light on the pathogenic origins of lupus; it may also pave the way for novel therapeutic approaches.

\section{Methods}

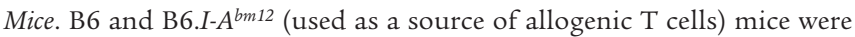
obtained from the Jackson Laboratory, and subsequently bred in our animal

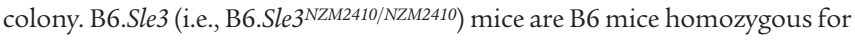
a 24-cM congenic interval (D7mit158-D7mit40) encompassing the NZM2410 allele of Sle3. Previous published reports had used a larger chromosome 7 interval encompassing Sle3 (and including Sle5), whereas the present study uses a recombinant offspring that includes Sle3 but excludes Sle5, as illustrated in Table 1. This novel strain retains the cellular phenotypes (i.e., 

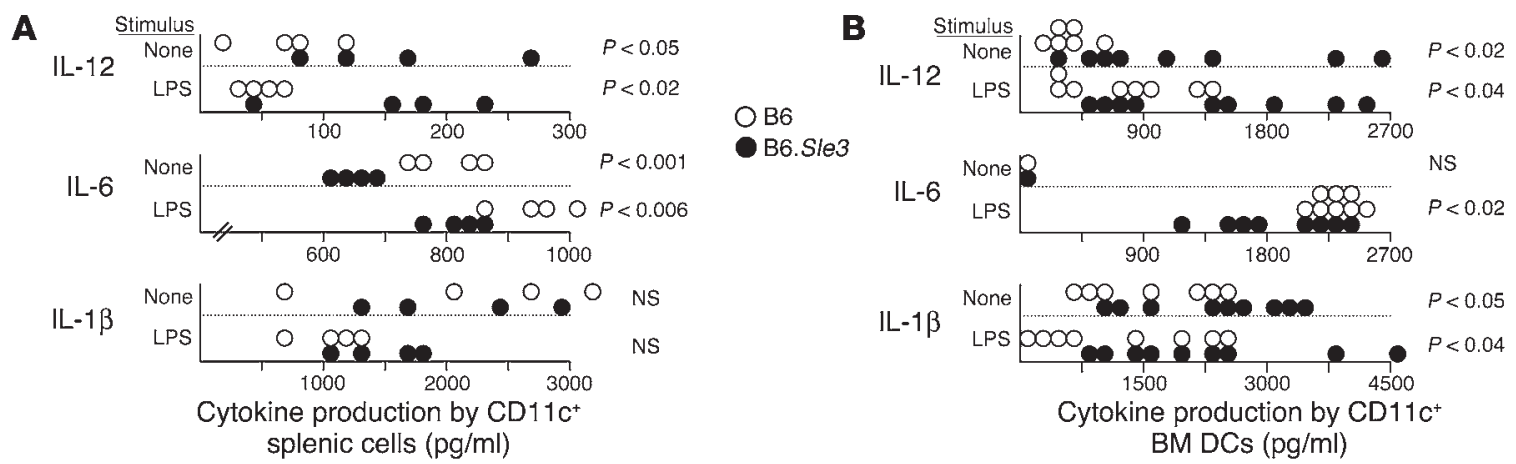

Cytokine production by CD11 $\mathrm{c}^{+}$ BM DCs (pg/ml)
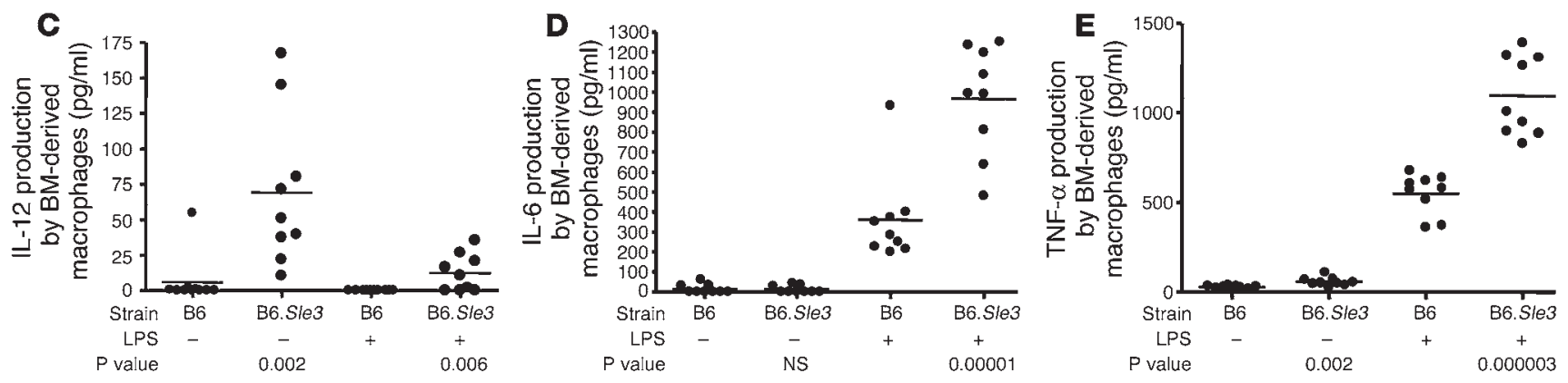

Figure 4

Cytokine secretion profiles of B6.Sle3 DCs and macrophages. (A and B) CD11c magnetic bead-purified splenic DCs from 2- to 3-month-old B6 and B6.Sle3 mice ( $n=4$ per group; A), as well as CD11c magnetic bead-purified DCs cultured from B6 or B6.Sle3 BM (using GM-CSF alone, for 7 days; $n=8-9$ mice per group; B), were incubated for 24 hours, with or without $10 \mathrm{ng} / \mathrm{ml} \mathrm{LPS}$. Depicted are the IL-12 (p70), IL-6, and IL-1 $\beta$ levels in 24-hour culture supernatant. Each dot represents data derived from splenic or BM DCs of an individual mouse; where the positions of the dots overlap, this has been represented using single dots only, for clarity. Similar findings were noted in a second confirmatory study (Supplemental Figure 1A). (C-E) In addition, 24-hour IL-12, IL-6, and TNF- $\alpha$ secretion by B6- or B6.Sle3-derived BM-cultured macrophages in response to LPS was assessed by ELISA. Each dot represents data derived from the BM macrophages of an individual mouse. The horizontal bars represent group means. The data shown were reproduced in 2-3 additional experiments (Supplemental Figure 1B). The depicted $P$ values in A-E were computed by comparison of the B6.Sle3 values with the B6 control values, using the Student's $t$ test.

elevated CD4/CD8 ratios and low-grade serological autoreactivity) noted previously in mice bearing the larger disease interval (23). B6.OT-II mice are B6 mice bearing an I-A $\mathrm{A}^{\mathrm{b}}$-restricted, OVA-specific TCR Tg (29), obtained as a kind gift from William Heath (Walter and Eliza Hall Institute, Parkville, Victoria, Australia). B6.OT-II mice and B6.Sle3 mice were interbred over 2 generations to obtain mice that were homozygous for Sle3, and positive for the OT-II TCR Tg; these mice are referred to as B6.Sle3.OT-II. The presence of the Sle 3 interval was ascertained by PCR using the microsatellite markers

\section{Figure 5}

Impaired apoptosis in B6.Sle3 macrophages and DCs. (A and B) DCs and macrophages were cultured from the BM of B6 and B6.Sle3 mice, and incubated without any stimuli for 24 hours. Following culture, the extent of apoptosis was gauged by flow cytometry using annexin $V$ and 7-amino-actinomycin D (7-AAD), after gating on CD11c-expressing DCs or $\mathrm{F} 4 / 80^{+}$macrophages. Each group and bar represent data gleaned from $6-7$ individual mice from each strain. $P$ values were computed by comparison of the B6.Sle3 values with the $\mathrm{B} 6$ control values, using the Student's $t$ test. The shown horizontal bars indicate the respective group means. (C and $\mathbf{D}$ ) In addition, DCs cultured from 2-month-old $\mathrm{B} 6$ or B6.Sle3 $\mathrm{BM}$ were pulsed with 1,000 nM OVA $323-339$, washed, and cocultured (10,000 per well) with OVA-specific B6.OT-II TCR Tg $T$ cells for varying times, as indicated. After culture, the fraction of $T$ cells that were apoptotic (based on expression of annexin V; C) and the mean fluorescence intensity (mfi) of CD69 on the $\mathrm{Tg} T$ cells (D) were assessed by flow cytometry. The respective $\mathrm{B} 6$ and $\mathrm{B} 6 . \mathrm{S} / \mathrm{e} 3$ values were compared using the Student's $t$ test $\left({ }^{*} P<0.05\right.$; $\left.{ }^{* *} P<0.001\right)$.
D7mit158,D7mit145,D7mit31, and D7mit40. The presence of the TCR Tg was screened by PCR using a TCR V $\beta 5$-specific primer, and confirmed by FACS, using Abs to TCR V $\beta 5$ and V $\alpha 2$. All mice used in this study were bred and housed in a specific pathogen-free colony, and these studies were approved
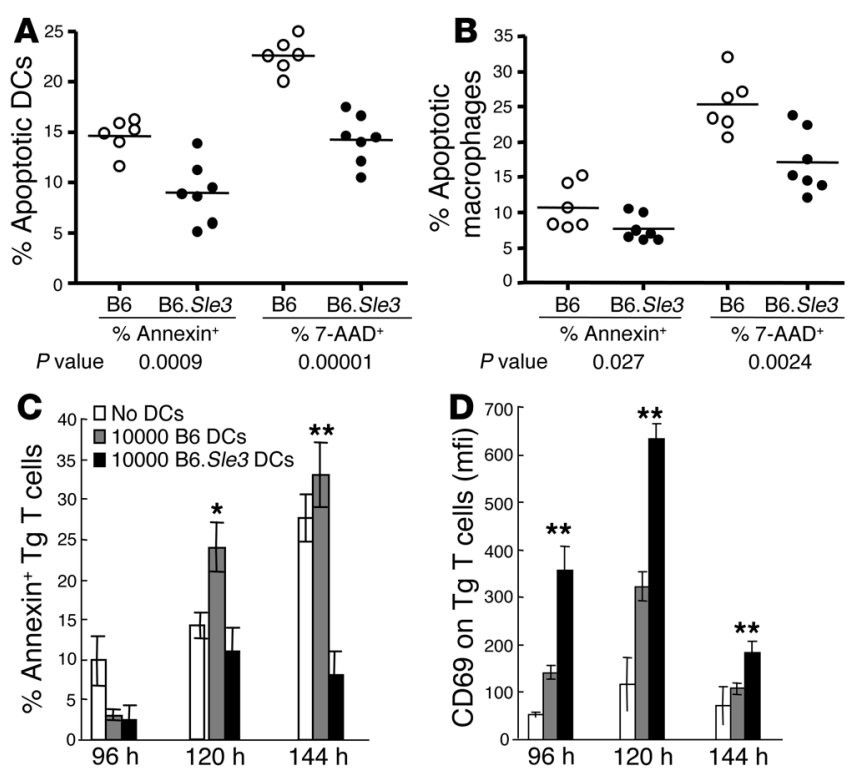

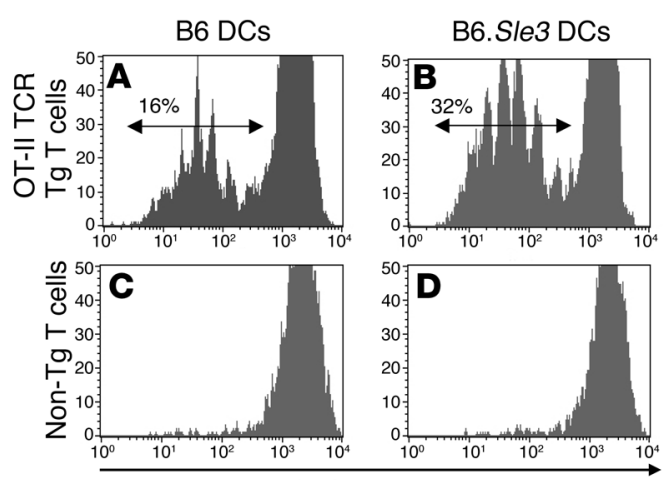

CFSE staining
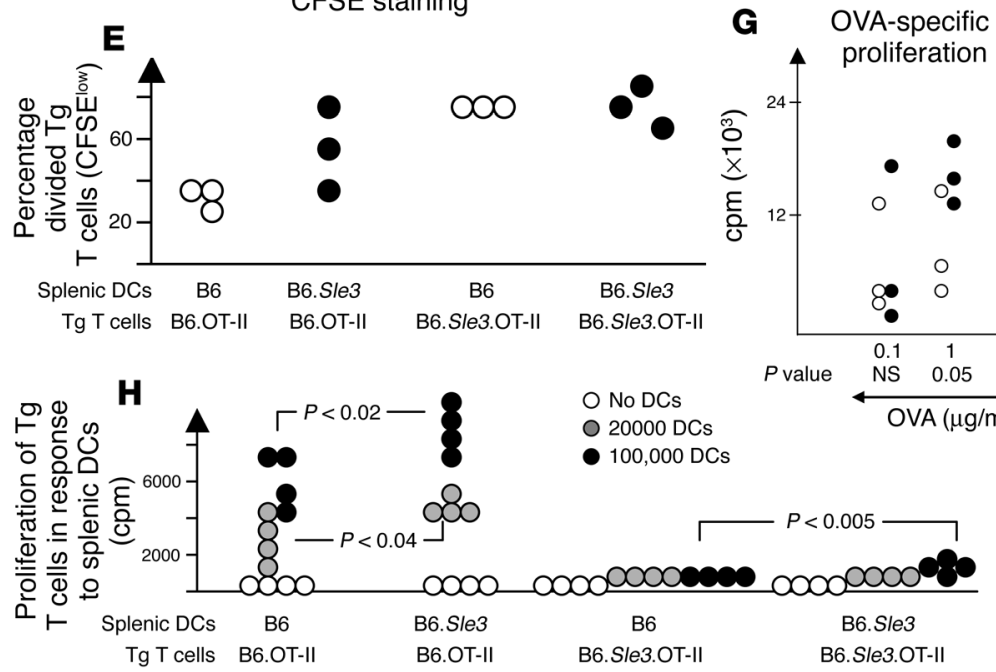

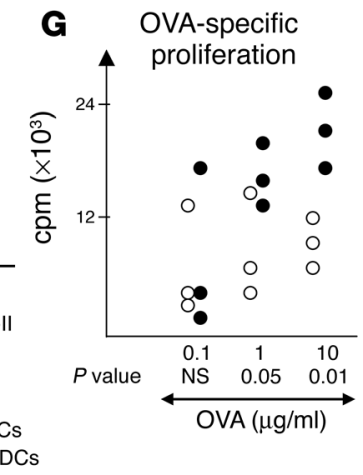

\section{F OVA-specific $\quad$ Figure 6}

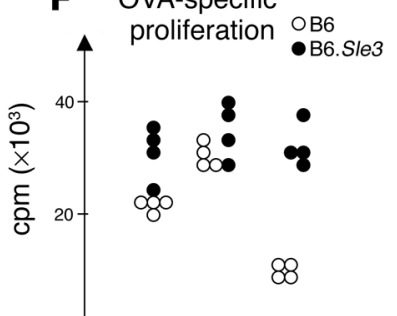

B6.Sle3 DCs are superior APCs for T cell stimulation. (A-D) DCs cultured from 2-month-old B6 (A and C) or B6.Sle3 (B and D) BM, using GM-CSF plus IL-4 for 7 days, were pulsed with $\mathrm{OVA}_{323-339}$, and cocultured with CFSE-labeled, OVA-specific B6.OT-II TCR Tg T cells ( $\mathbf{A}$ and $\mathbf{B}$ ) or non-Tg T cells ( $\mathbf{C}$ and $\mathbf{D})$, and the fraction of $T$ cells that had undergone cell division was assessed by flow cytometry. The histograms pertain to coculture studies that were performed with 10,000 OVA-pulsed DCs, and examined by flow cytometry 96 hours after stimulation. (E) Similar results were obtained using OVA-pulsed CD11c bead-purified splenic DCs from B6 and B6.Sle3 mice cocultured with B6.OT-II or B6.Sle3.OT-II T cells for 96 hours; however, any apparent differences noted failed to attain statistical significance. The data depicted in $\mathbf{E}$ are representative of 3 independent experiments. In addition, OVA-specific B6.OT-II TCR Tg T cells were cocultured with varying numbers of unpulsed $\mathrm{B} 6$ (white dots) or B6.Sle3 (black dots) BM-cultured DCs and 1,000 nM $\mathrm{OVA}_{323-339}(\mathbf{F})$, or with 50,000 unpulsed BM-derived DCs and varying doses of $\mathrm{OVA}_{323-339}(\mathbf{G})$. In both experiments, proliferation was assessed 96 hours after culture, by assaying of ${ }^{3} \mathrm{H}$-thymidine incorporation. The data portrayed in $\mathbf{F}$ and $\mathbf{G}$ are representative of 3 independent experiments; an additional experiment is displayed in $\mathbf{H}$, where the proliferation of B6.OT-II and B6.Sle3.OT-II TCR Tg T cells in response to varying numbers of OVA-pulsed B6 or B6.Sle3 splenic CD11C bead-purified DCs was assessed. Shown $P$ values were computed by comparison of the B6.Sle3 values with the B6 control values, using the Student's $t$ test. by the University of Texas Southwestern Medical Center institutional review board. Both male and female mice were used in equal numbers, since no sex differences in phenotype were observed.

Cell isolation and coculture studies. Spleens and inguinal lymph nodes were cut into small fragments, and then digested with collagenase D $(1 \mathrm{mg} / \mathrm{ml}$; Sigma-Aldrich) and DNase I (0.02 mg/ml; Roche Diagnostics Corp.) in
RPMI 1640 medium supplemented with $5 \% \mathrm{FCS}$, for 20 minutes at $37^{\circ} \mathrm{C}$. Digested fragments were washed twice in PBS $/ 2 \%$ FCS $/ 5$ mM EDTA, and single-cell suspensions were made. For the functional studies, splenic $\mathrm{T}$ cells were purified using anti-CD4 magnetic beads (Miltenyi Biotec) and were more than $95 \%$ pure. For the $\mathrm{T}$ cell proliferation assays, whole splenocytes or splenic $\mathrm{T}$ cells were stimulated with the following I-A $\mathrm{A}^{\mathrm{b}}$-binding peptides:
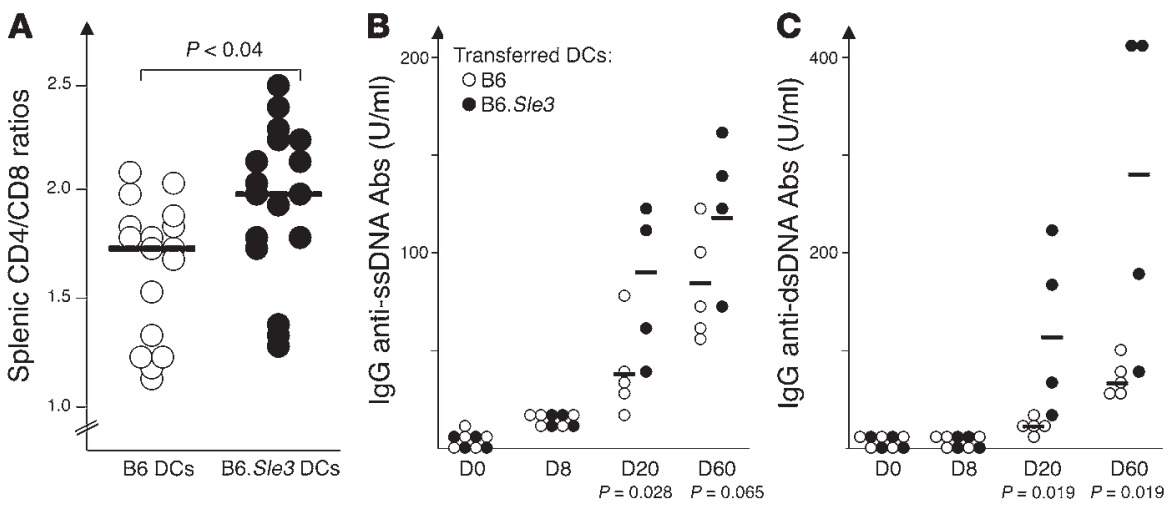

Figure 7

Adoptively transferred B6.Sle3 DCs recreate Sle3-associated phenotypes. DCs cultured from B6 or B6.Sle3 BM (16-18 mice per strain, pooled from 4 independent experiments) were adoptively transferred into 2-month-old B6 mice on D0, D7, and D14, with coadministration of LPS on D1, D8, and D15. Plotted in A are the terminal splenic CD4/CD8 ratios in the host mice on D20 or D60; since the outcomes were similar at both time points, the results from D20 and D60 experiments have been pooled. Plotted in B are the serum IgG anti-single-stranded DNA Abs (left) and IgG anti-doublestranded DNA Abs (right) at serial time points during the 60-day study period, after the transfer of B6 or B6.S/e3 DCs, with coadministered LPS. All $P$ values shown were computed by comparison of the B6.Sle3 values with the B6 control values, using the Student's $t$ test. The horizontal bars indicate the respective group means. The data shown were reproduced in 2 additional studies, as portrayed in Supplemental Figure 2. 
I-E $\boldsymbol{\alpha}_{77-93}$ (ASFEAQGALANIAVDKA), histone H476-90 (AKRKTVTAMDVVYAL), and OVA $323-339$ (ISQAVHAAHAEINEAGR), synthesized at the University of Texas Southwestern protein core facility. For the T:APC coculture studies, splenic T cells $\left(5 \times 10^{5} / \mathrm{ml}\right)$ purified with anti-CD4 beads, and splenic or BM-derived DCs $\left(5 \times 10^{5} / \mathrm{ml}\right.$, or as indicated $)$ irradiated with 30 Gy and pulsed with OVA (1,000 nM peptide, unless otherwise indicated), were cocultured for 24-108 hours, and the T cell response was ascertained by measurement of thymidine incorporation, as described previously (23). In addition, IFN- $\gamma$ and IL-4 production was assessed using commercially available kits (R\&D Systems). In a variation of this assay, the T:DC coculture assay was performed using splenic $\mathrm{T}$ cells that were labeled with CFSE (Invitrogen Corp.), and the degree of cell division was gauged from the serial diminution of the CFSE label, as determined by flow cytometry.

Flow cytometric analysis and Abs. Flow cytometric analysis was performed as described previously $(23,25,27)$. Briefly, cells were first blocked with staining medium (PBS, $5 \%$ horse serum, $0.05 \%$ azide) containing $10 \%$ normal rabbit serum. Cells were then stained on ice with optimal amounts of FITC-, PE-, or biotin-conjugated primary Abs diluted in staining medium for 30 minutes. The following dye- or biotin-coupled Abs were obtained from BD Biosciences - Pharmingen, or CALTAG Laboratories, and used at pretitrated dilutions: CD11b (M1/70), CD11c (HL3), Gr-1 (RB6-8C5), F4/80 (RM2905), Vß5 (MR9-4), Vo2 (B20.1), CD40 (3/23), CD54/ICAM-1 (3E2), CD106/VCAM-1 (429A), CD16/32 (2.4G2), CD4 (RM4-5), CD5 (53-7.3), CD8 (Ly-2), CD23 (B3B4), CD25 (7D4), CD43 (S7), CD44 (IM7), CD45R/B220 (RA3-6B2), CD62L (MEL14), CD69 (H1.2F3), CD80/B7-1 (16-10A1), and CD86/B7-2 (GL1). Whereas the Abs to CD11b, CD11c, Gr-1, CD8, B220, and F4/80 were used to define different cell subsets, the levels of CD80, CD86, CD40, FcR, $\mathrm{CD} 54, \mathrm{CD} 106$, and I- $\mathrm{A}^{\mathrm{b}}$ were used to gauge the activation/maturation status of these cells. Cell staining was analyzed using a FACScan (BD). For the data depicted in Tables 2 and 3, and in Figure 3, all isotype-control staining yielded median fluorescence intensities of less than 60 units, with the exception of FITC-coupled hamster IgG (used as a control for FITC-coupled antiCD54), which stained macrophages (but not other cells) somewhat more strongly (mean and median fluorescence intensities were 134 and 104 units, respectively). Dead cells were excluded on the basis of scatter characteristics, and 20,000-50,000 events were acquired per sample.

Serology and pathology. The anti-double-stranded DNA, anti-singlestranded DNA, and anti-histone/DNA ELISAs were carried out as described before $(22,25,27)$. Raw OD was converted to $\mathrm{U} / \mathrm{ml}$, using a positive control serum derived from a B6.Sle1.FAS ${ }^{l p r}$ mouse (27), arbitrarily setting the reactivity of a 1:100 dilution of this serum to $100 \mathrm{U} / \mathrm{ml}$. Renal disease was assessed as described previously $(25,27)$.

$D C /$ macrophage culture and functional studies. Single-cell suspensions of BM were cultured with M-CSF, or IL-4 plus GM-CSF, or GM-CSF alone (10 ng/ $\mathrm{ml}$ for all cytokines; R\&D Systems). Low-endotoxin FBS (HyClone) was used, and endotoxin contamination of culture medium was excluded using the LAL QCL-1000 kit from BioWhittaker Inc. Seven days after culture, the composition of the cultured cells was ascertained by flow cytometry, using Abs to $\mathrm{CD} 11 \mathrm{~b}, \mathrm{CD} 11 \mathrm{c}, \mathrm{F} 4 / 80$, and Gr-1. Cultured cells were then enumerated and used for various in vitro or in vivo studies, as indicated. For the functional assays, BM-derived macrophages or DCs were cultured at $10^{6} \mathrm{cells} / \mathrm{ml}$, and stimulated with the indicated doses of LPS. In vitro IL-1 $\beta$, IL-6, TNF- $\alpha$, and IL-12 p70 production was measured using commercial ELISA kits (R\&D Systems). For some experiments, CD $11 \mathrm{c}^{+} \mathrm{DCs}$ were purified using CD $11 \mathrm{c} \mathrm{mag-}$ netic beads (either from freshly isolated spleens or from GM-CSF-driven BM cultures), following the manufacturer's instructions (Miltenyi Biotec), before use in functional studies. For gauging the degree of apoptosis, BM-cultured macrophages or DCs were cultured with or without LPS $(10 \mathrm{ng} / \mathrm{ml})$ for the indicated time periods, after which the cells were stained with annexin $\mathrm{V}$ (BD Biosciences - Pharmingen) and 7-amino-actinomycin D (7-AAD; EMD Biosciences) and examined by flow cytometry.

In vivo studies. For the in vivo antigen response experiments, $10 \times 10^{6}$ B6.OT-II splenic TCR Tg T cells were transferred i.v. into 2-month-old B6 or B6.Sle3 mice on D0, challenged with OVA (100 $\mu$ g i.p.) in complete Freund's adjuvant on D1, and examined for serological and splenic cellular phenotypes on D7. For the peripheral tolerance studies, 2 sets of experiments were conducted. In 1 experiment, OVA was administered i.p. (100 $\mu \mathrm{g} /$ mouse) in incomplete Freund's adjuvant (Figure 2C). A second panel of mice were injected with OVA in incomplete Freund's adjuvant on D0 (100 $\mu \mathrm{g} /$ mouse), and subsequently with OVA in complete Freund's adjuvant on D10 (100 $\mu \mathrm{g} /$ mouse) (Figure 2D). With both sets of mice, the proliferative response of splenic T cells to OVA was assessed 5 days after the last injection of OVA. For the adoptive transfer experiments, BM-derived DCs (cultured using IL-4 plus GM-CSF, as detailed above) from B6 or B6.Sle3 mice were transferred i.v. into 2-month-old B6 or B6.OT-II recipients (3 injections of $10^{7}$ cells per mouse, spaced at weekly intervals). One day after each DC transfer, the recipient mice were administered LPS (30 $\mu$ g i.p.). At the indicated times after transfer, serum autoantibodies were assayed by ELISA, and the phenotype of splenocytes was studied by flow cytometry.

Statistics. Where the samples studied were normally distributed, statistical comparisons were performed using the Student's $t$ test; otherwise, the Mann-Whitney $U$ test was used. These tests were executed using Excel (Microsoft Corp.) or SigmaStat (Jandel Scientific).

\section{Acknowledgments}

Work in the authors' laboratory is funded by grants from the NIH (AR44894, AR51642, and AR50812) and the National Arthritis Foundation. We would also like to acknowledge Borna Mehrad for helpful feedback, and Bill Heath (WEHI) for the generous gift of B6.OT-II mice.

Received for publication August 16, 2004, and accepted in revised form May 9, 2005.

Address correspondence to: Chandra Mohan, Simmons Arthritis Research Center, Department of Internal Medicine/Rheumatology, University of Texas Southwestern Medical Center, Mail Code 8884, Y8.204, 5323 Harry Hines Boulevard, Dallas, Texas 753908884, USA. Phone: (214) 648-9675; Fax: (214) 648-7995; E-mail: Chandra.mohan@utsouthwestern.edu.

JianKun Zhu and XueBin Liu contributed equally to this work.
1. Hahn, B.H., and Datta, S.K. 1997. Characteristics of T-cells that participate in the pathogenesis of SLE. Lupus. 6:330-332.

2. Craft, J., Peng, S., Fujii, T., Okada, M., and Fatenejad, S. 1999. Autoreactive T cells in murine lupus: origins and roles in autoantibody production. Immunol. Res. 19:245-257.

3. Chen, S.Y., et al. 1996. The natural history of disease expression in CD4 and CD8 gene-deleted New Zealand black (NZB) mice. J. Immunol.
157:2676-2684.

4. Hang, L., Theofilopoulos, A.N., Balderas, R.S., Francis, S.J., and Dixon, F.J. 1984. The effect of thymectomy on lupus-prone mice. J. Immunol. 132:1809-1813.

5. Koh, D.R., et al. 1995. Murine lupus in MRL/lpr mice lacking CD4 or CD8 T cells. Eur. J. Immunol. 25:2558-2562.

6. Steinberg, A.D., Law, L.D., and Talal, N. 1970. The role of NZB-NZW F1 thymus in experimental tolerance and auto-immunity. Arthritis Rheum. 13:369-377.

7. Wofsy, D., and Seaman, W.E. 1987. Reversal of advanced murine lupus in NZB/NZW F1 mice by treatment with monoclonal antibody to L3T4. J. Immunol. 138:3247-3253.

8. Ando, D.G., Sercarz, E.E., and Hahn, B.H. 1987. Mechanisms of $\mathrm{T}$ and $\mathrm{B}$ cell collaboration in the in vitro production of anti-DNA antibodies in the NZB/NZW F1 murine SLE model. J. Immunol. 138:3185-3190. 
9. Datta, S.K., Kaliyaperumal, A., Mohan, C., and Desai-Mehta, A. 1997. T helper cells driving pathogenic anti-DNA autoantibody production in lupus: nucleosomal epitopes and CD40 ligand signals. Lupus. 6:333-336.

10. Singh, R.R., et al. 1995. T cell determinants from autoantibodies to DNA can upregulate autoimmunity in murine systemic lupus erythematosus. J. Exp. Med. 181:2017-2027.

11. Peng, S.L., and Craft, J. 1996. T cells in murine lupus: propagation and regulation of disease. Mol. Biol. Rep. 23:247-251.

12. Diaz Gallo, C., et al. 1992. Autoreactive kidneyinfiltrating T-cell clones in murine lupus nephritis. Kidney Int. 42:851-859.

13. Yamada, M., et al. 2002. Selective accumulation of CCR4+ T lymphocytes into renal tissue of patients with lupus nephritis. Arthritis Rheum. 46:735-740.

14. Kono, D.H., and Theofilopoulos, A.N. 2000. Genetics of systemic autoimmunity in mouse models of lupus. Int. Rev. Immunol. 19:367-387.

15. Vyse, T.J., and Kotzin, B.L. 1998. Genetic susceptibility to systemic lupus erythematosus [review]. Annu. Rev. Immunol. 16:261-292.

16. Wakeland, E.K., Wandstrat, A., Liu, K., and Morel, L. 2000. Genetic dissection of systemic lupus erythematosus. Curr. Opin. Immunol. 11:701-707.

17. Morel, L., Rudofsky, U.H., Longmate, J.A., Schiffenbauer, J., and Wakeland, E.K. 1994. Polygenic control of susceptibility to murine systemic lupus erythematosus. Immunity. 1:219-229.

18. Kono, D.H., et al. 1994. Lupus susceptibility loci in New Zealand mice. Proc. Natl. Acad. Sci. U. S. A. 91:10168-10172.

19. Drake, C.G., et al. 1995. Analysis of the New Zealand Black contribution to lupus-like renal disease. Multiple genes that operate in a threshold manner. J. Immunol. 154:2441-2447.

20. Vidal, S., Kono, D.H., and Theofilopoulos, A.N. 1998. Loci predisposing to autoimmunity in MRLFas Ipr and C57BL/6-Faslpr mice. J. Clin. Invest. 101:696-702.

21. Santiago, M.L., et al. 1998. Linkage of a major quantitative trait locus to Yaa gene-induced lupuslike nephritis in (NZW x C57BL/6)F1 mice. Eur. J. Immunol. 28:4257-4267.

22. Morel, L., et al. 1997. Functional dissection of SLE pathogenesis using congenic mouse strains. J. Immunol. 158:6019-6028.

23. Mohan, C., Yu, Y., Morel, L., Yang, P., and Wakeland, E.K. 1999. Genetic dissection of SLE pathogenesis: Sle 3 on murine chromosome 7 impacts $\mathrm{T}$ cell activation, differentiation and cell death. J. Immunol. 162:6492-6502.

24. Wakui, M., Kim, J., Butfiloski, E.J., Morel, L., and Sobel, E.S. 2004. Genetic dissection of lupus pathogenesis: Sle $3 / 5$ impacts IgH CDR3 sequences, somatic mutations, and receptor editing. J. Immunol. 173:7368-7376.

25. Mohan, C., et al. 1999. Genetic dissection of SLE pathogenesis: a recipe for nephrophilic autoantibodies. J. Clin. Invest. 103:1685-1695.

26. Morel, L., et al. 2000. Genetic reconstitution of systemic lupus erythematosus immunopathology with polycongenic murine strains. Proc. Natl. Acad. Sci.U.S. A. 97:6670-6675.

27. Shi, X., Xie, C., Kreska, D., Richardson, J.A., and Mohan, C. 2002. Genetic dissection of SLE: SLE1 and FAS impact alternate pathways leading to lymphoproliferative autoimmunity. J. Exp. Med. 196:281-292.

28. Sobel, E.S., et al. 2002. Genetic dissection of SLE pathogenesis: evidence for functional expression of Sle3/5 in non-T-cells. J. Immunol. 169:4025-4032.

29. Barnden, M.J., Allison, J., Heath, W.R., and Carbone, F.R. 1998. Defective TCR expression in transgenic mice constructed using cDNA-based $\alpha$ - and $\beta$-chain genes under the control of heterologous regulatory elements. Immunol. Cell Biol. 76:34-40.

30. Wofsy, D., Kerger, C.E., and Seaman, W.E. 1984 Monocytosis in the BXSB model for systemic lupus erythematosus. J. Exp. Med. 159:629-634.

31. Vieten, G., et al. 1992. Expanded macrophage precursor populations in BXSB mice: possible reason for the increasing monocytosis in male mice. Clin. Immunol. Immunopathol. 65:212-218.

32. Vieten, G., Grams, B., Muller, M., Hartung, K. and Emmendorffer, A. 1996. Examination of the mononuclear phagocyte system in lupus-prone male BXSB mice. J. Leukoc. Biol. 59:325-332.

33. Muller, M., Emmendorffer, A., and Lohmann-Matthes, M.L. 1991. Expansion and high proliferative potential of the macrophage system throughout life time of lupus-prone NZB/W and MRL lpr/lpr mice. Lack of down-regulation of extramedulla macrophage proliferation in the postnatal period. Eur. J. Immunol. 21:2211-2217.

34. Russell, P.J., Cahill, J., Cameron, F., and Hume, D. 1985. Studies of peritoneal macrophage function in murine systemic lupus erythematosus. II. Nature of elevated resident peritoneal cells in NZB and (NZB x NZW)F1 mice. J. Leukoc. Biol. 38:241-254.

35. Levine, J., Hartwell, D., and Beller, D.I. 1991. Imbalanced cytokine production by macrophages from autoimmune-prone mice. Immunol. Lett. 30:183-192.

36. Ishikawa, S., et al. 2002. Increased circulating $\mathrm{CD} 11 \mathrm{~b}+\mathrm{CD} 11 \mathrm{c}+$ dendritic cells (DC) in aged BWF1 mice which can be matured by TNF-alpha into BLC/CXCL13-producing DC. Eur. J. Immunol. 32:1881-1887

37. Ishikawa, S., et al. 2001. Aberrant high expression of B lymphocyte chemokine (BLC/CXCL13) by $\mathrm{C} 11 \mathrm{~b}+\mathrm{CD} 11 \mathrm{c}+$ dendritic cells in murine lupus and preferential chemotaxis of B1 cells towards BLC. J. Exp. Med. 193:1393-1402.

38. Adachi, Y., et al. 2002. Marked increase in number of dendritic cells in autoimmune-prone (NZW $x$ BXSB)F1 mice with age. Stem Cells. 20:61-72.

39. Vasquez, A.C., Feili-Hariri, M., Tan, R.J., and Morel, P.A. 2004. Qualitative and quantitative abnormalities in splenic dendritic cell populations in NOD mice. Clin. Exp. Immunol. 135:209-218.

40. Jun, H.S., Yoon, C.S., Zbytnuik, L., van Rooijen, N., and Yoon, J.W. 1999. The role of macrophages in $\mathrm{T}$ cell-mediated autoimmune diabetes in nonobese diabetic mice. J. Exp. Med. 189:347-358.

41. Marleau, A.M., and Singh, B. 2002. Myeloid dendritic cells in non-obese diabetic mice have elevated costimulatory and $\mathrm{T}$ helper-1-inducing abilities. J. Autoimmun. 19:23-35.

42. Poligone, B., Weaver, D.J., Jr., Sen, P., Baldwin, A.S., Jr., and Tisch, R. 2002. Elevated NF-kappaB activation in nonobese diabetic mouse dendritic cells results in enhanced APC function. J. Immunol. 168:188-196.

43. Harder, K.W., et al. 2001. Gain- and loss-of-function Lyn mutant mice define a critical inhibitory role for Lyn in the myeloid lineage. Immunity. 15:603-615.

44. Jiao, H., et al. 1997. Macrophages from motheaten and viable motheaten mutant mice show increased proliferative responses to GM-CSF: detection of potential HCP substrates in GM-CSF signal transduction. Exp. Hematol. 25:592-600.

45. Barclay, A.N., Wright, G.J., Brooke, G., and Brown, M.H. 2002. CD200 and membrane protein interactions in the control of myeloid cells. Trends Immunol. 23:285-290.

46. Weih, F., et al. 1996. Both multiorgan inflammation and myeloid hyperplasia in RelB-deficient mice are T cell dependent. J. Immunol. 157:3974-3979.

47. Ammon, C., Mondal, K., Andreesen, R., and Krause, S.W. 2004. Cutting edge: NF-kappa B2 is a negative regulator of dendritic cell function. J. Immunol. 172:752-756
48. Garza, K.M., et al. 2000. Role of antigen-presenting cells in mediating tolerance and autoimmunity. J. Exp. Med. 191:2021-2027.

49. Kinoshita, K., et al. 2000. Costimulation by B7-1 and B7-2 is required for autoimmune disease in MRL-Faslpr mice. J. Immunol. 164:6046-6056.

50. Mohan, C., Shi, Y., Laman, J.D., and Datta, S.K. 1995. Interaction between CD 40 and its ligand gp39 in the development of murine lupus nephritis. J. Immunol. 154:1470-1480.

51. Daikh, D.I., Finck, B.K., Linsley, P.S., Hollenbaugh, D., and Wofsy, D. 1997. Long-term inhibition of murine lupus by brief simultaneous blockade of the B7/CD28 and CD40/gp39 costimulation pathways. J. Immunol. 159:3104-3108.

52. Teague, T.K., Marrack, P., Kappler, J.W., and Vella, A.T. 1997. IL-6 rescues resting mouse T cells from apoptosis. J. Immunol. 158:5791-5796.

53. Curtsinger, J.M., et al. 1999. Inflammatory cytokines provide a third signal for activation of naive CD4+ and CD8+ T cells. J. Immunol. 162:3256-3262.

54. Maxwell, J.R., Weinberg, A., Prell, R.A., and Vella, A.T. 2000. Danger and OX40 receptor signaling synergize to enhance memory $\mathrm{T}$ cell survival by inhibiting peripheral deletion. J. Immunol. 164:107-112.

55. Maxwell, J.R., Ruby, C., Kerkvliet, N.I., and Vella, A.T. 2002. Contrasting the roles of costimulation and the natural adjuvant lipopolysaccharide during the induction of $\mathrm{T}$ cell immunity. J. Immunol. 168:4372-4381.

56. Vella, A.T., et al. 1997. CD28 engagement and proinflammatory cytokines contribute to $\mathrm{T}$ cell expansion and long-term survival in vivo. J. Immunol. 158:4714-4720.

57. Tang, T.T., et al. 1997. A role for Mac-1 (CD11b/CD18) in FcgR interactions in vivo: Mac-1 deficiency abrogates sustained neutrophil adhesion and proteinuria in Fc-dependent anti-glomerular basement membrane nephritis. J. Exp. Med. 186:1853-1863.

58. Westerhuis, R., et al. 2000. Distinctive roles of neutrophils and monocytes in anti-thy- 1 nephritis. Am. J. Pathol. 156:303-310.

59. Kong, P.L., Morel, L., Croker, B.P., and Craft, J. 2004. The centromeric region of chromosome 7 from MRL mice (Lmb3) is an epistatic modifier of Fas for autoimmune disease expression. J. Immunol. 172:2785-2794

60. Kovacs, B., Vassilopoulos, D., Vogelgesang, S.A., and Tsokos, G.C. 1996. Defective CD3-mediated cell death in activated $T$ cells from patients with systemic lupus erythematosus. Clin. Immunol. Immunopathol. 81:293-302.

61. Pascual, V., Banchereau, J., and Palucka, A.K. 2003. The central role of dendritic cells and interferonalpha in SLE. Curr. Opin. Rheumatol. 15:548-556.

62. Boswell, J., and Schur, P.H. 1989. Monocyte function in systemic lupus erythematosus. Clin. Immunol. Immunopathol. 52:271-278.

63. Kavai, M., et al. 1983. Signals of monocyte activation in patients with SLE. Clin. Exp. Immunol. 51:255-260.

64. Steinbach, F., et al. 2000. Monocytes from systemic lupus erythematous patients are severely altered in phenotype and lineage flexibility. Ann. Rheum. Dis. 59:283-288.

65. Krishnan, S., Farber, D.L., and Tsokos, G.C. 2003. $\mathrm{T}$ cell rewiring in differentiation and disease. J. Immunol. 171:3325-3331.

66. Tsokos, G.C., Nambiar, M.P., Tenbrock, K., and Juang, Y.-T. 2003. Rewiring the T-cell: signaling defects and novel prospects for the treatment of SLE [review]. Trends Immunol. 24:259-263.

67. Krishnan, S., et al. 2004. Alterations in lipid raft composition and dynamics contribute to abnormal $\mathrm{T}$ cell responses in systemic lupus erythematosus. J. Immunol. 172:7821-7831. 